\title{
A step toward a standardized guide in sailing the sea: the Pilot's book digital upgrade
}

\author{
Roberto Manuzzo $^{a}$, Sonja Grosso $^{a}$, Riccardo Ansaldi $^{a}$, Ludovico Sturla $^{\mathrm{a}}$, C.C. Marta Pratellesi ${ }^{\mathrm{a}}$ \\ - Italian Hydrographic Institute (IIM), Genoa (ITALY) \\ * Corresponding author: ludovico_sturla@marina.difesa.it
}

\section{Keywords: Pilot's Book, digital upgrade, geotagged nautical information, safety of life at sea}

If compared to traditional charts, the Pilot's Book (hereafter PB, also known as Sailing directions or Rutter in its ancient form) provides complementary information. Therefore, the PB is necessary to sailors and mariners to face with all the natural and artificial entities their ships approach along their routes. Starting from the seventies, the role of the PB publications has been officialised by the International Convention for the Safety of Life at Sea (SOLAS, 1974) as one of the mandatory official nautical publications to be held on-board the SOLAS ships and carefully updated.

To date, whereas others official nautical publications have been already converted to - and therefore benefit from the advent of - the digital supports (personal computers, smartphones, the Electronic Chart Display and Information System or ECDIS, etc....), the PB remains one of the few publications lacking for an International Hydrographic Organization (IHO) standard guiding the Govemment Authorities how to provide PBs in digital formats. So, official PBs are mainly provided in traditional forms. This digital backwardness limits both the organization, the consultation and the updating of the information contained in the PBs. From the Govemment Authorities point of view, the collection (see the cyan box in Figure 1) and the organization of geo-referred information (yellow box) has to adapt to the paper book skeleton (chapters, sections, subsections and so on), often hiding the true relationships between the PB contents in a jungle of references and margin notes. Furthermore, the information in some PBs is distributed assuming a reasonable ship route to be followed by the sailor, which is clearly different from the infinite multitude of possible routes followed in real cases, when the sea and weather conditions force the sailors to take economic- and/or safety-driven decisions. So, it is clear why the interesting information is often scattered throughout the entire PB, implying a not-so-reasonable time to be spent by the sailor in acquiring the needed knowledge for safety to be guaranteed. Last but not least, the updating process of the information contained in the PB requires a relatively long time period for information to be collected and broadcasted (see all the boxes of Figure 1 but the red one) via the Notices to Mariners published by the Authorities and to be received and included in the PB on-board the ships by the mariners.



Figure 1. Workflow for the PBs to be collected (cyan box), manipulated (yellow box) and rendered to the mariners (green box). The digitization of the broadcasting process (from yellow to green boxes) and the rendering of PBs content in digital forms (red box) are the objectives of the present work.

In the present work, we show how the Italian Hydrographic Institute (IIM) is upgrading the PB to its digital version. The upgrade aims at solving most of the aforementioned problems, that is, 1) to clarify and to make explicit the relations between each of the PB elements (therefore minimizing the time spent at collecting and understanding their interplays), 2) to allow human-style and/or geotagged points queries (in order the sailor to know instantaneously the information worth knowing about the planned route) and 3) to optimize the updating process of the PB content. 
The first step to digitize the traditional PB information is to classify its content in a relational database management system (e.g. SQLserver or ORACLE). The classification requires the formalization both of the objects (e.g. harbours, authorities, winds, dangers, restrictions, regulations, weather conditions and so on) and the relations between these objects (the relations being hidden in the traditional PB structure).

Secondly, each object has to be geotagged by a Region of Interest (hereafter called ROI) which can be either the - possibly scaled up - extension of the object itself (in case of extended objects such as prohibited zones or maritime areas) or a circle centred on the object, the radius of which represents a "reasonable" (or fuzzy) area around the object (in case of point-like entities). The scale-up factor to magnify the extended object ROIs and the radius of circles centred on the pointlike objects are functions of the kinematics of the ship (its velocity) in order the ship crew to be properly forewarned. At a given time, information worth knowing is selected by intersecting the ship position with the ROIs of each object contained in the PB database. The data associated to each of the selected objects are then rendered in plain language. Therefore, ad-personam PBs can be demanded by sailors once the ship route or the geographical zones of interest are known. Figure 2 shows an example to understand the principles underneath the selection of information. The ship positions (the blue dashed line drawn on the chart in the left part of the figure) is compared to the ROIs of the elements contained in some region (here we show a chart only for practical purposes: in fact the ship route is compared to the positions and ROIs of the PB objects, these objects being located in real space as if they would be charted). The ROIs crossed by the ship route (green ROIs) indicate the object worth selecting, whereas ROIs not crossed (red ones) are ignored. In the database (centre of the figure), objects are coded as a collection of metadata. Each object contains also the position and the extension of its ROI. The green and the red arrows points respectively to the selected and not selected objects. The information associated to the selected objects (which are relevant to navigation safety) is then collected in sharable document formats (pdf or html files) both being simply reported or being wrapped by conventional texts (respectively sections 1.2.2 and 1.2.1 in the right part of the figure).
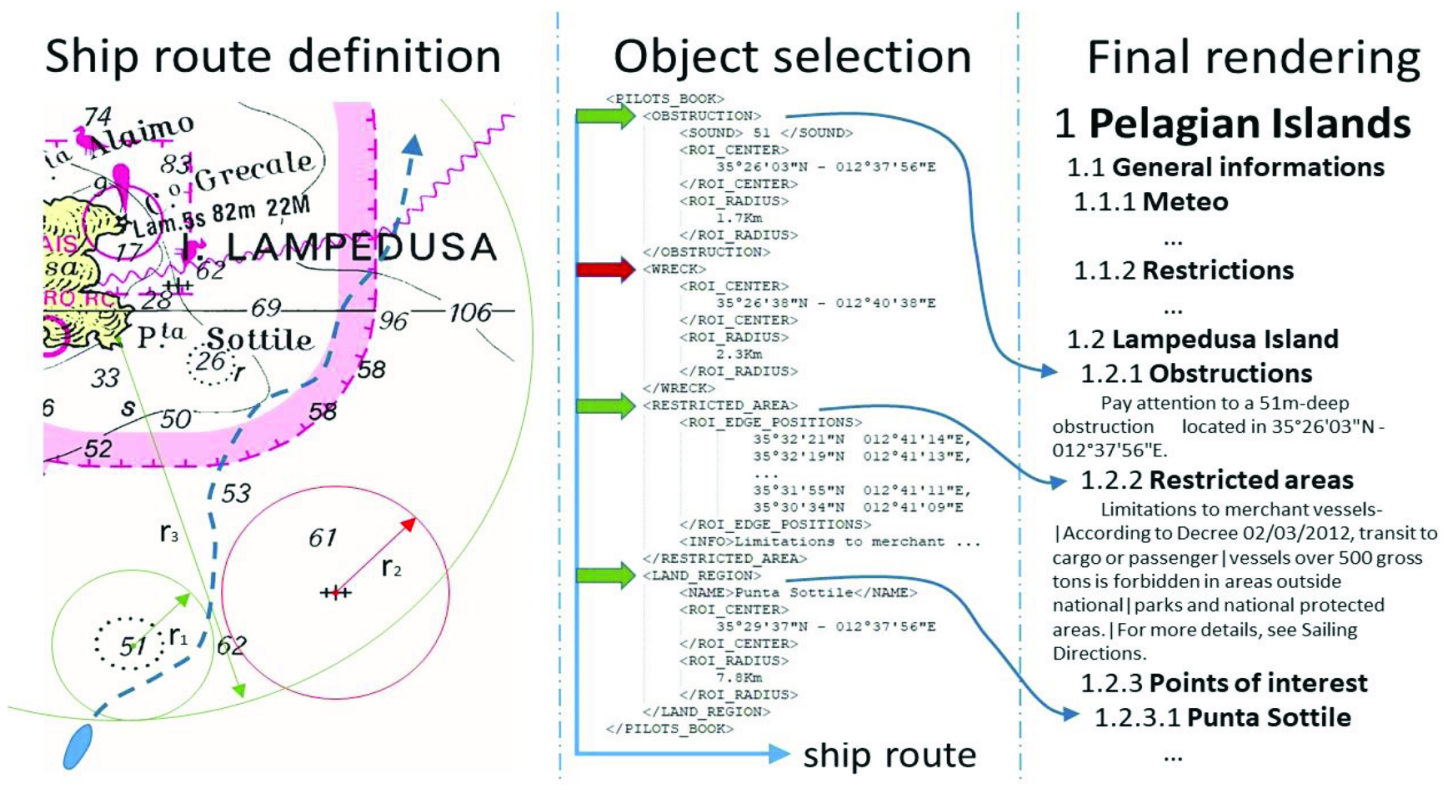

Figure 2. Example of a personalized PB compilation starting from a ship route plan (see text for details). In the figure we observe: (left side) a chart where PB objects and the associated ROIs have been drawn, (centre) the database collection of metadata associated to some of the charted objects and the selection process depending by the ship route (green and red arrows) and (right side) the rendering of the selected objects information in a personalized PB chapter.

Both from the author and the reader perspectives, there is no direct access to the database content. The creation, the modification, the deletion, the consultation and the extraction of the database contents are mediated by a Graphical User Interface which can be an executable, portable and multi-platform PC program or a web-based interface. This interface mediates the database content manipulations by means of eXtensible Markup Language (XML) files that allows rendering masks (XSLT and Latex) to be applied to the selected database contents. So, the selected content can be rendered depending by the final user, this being the sailor (yachting sector), the mariner or, thinking about the S-100 perpectives, the ECDIS (for human driven and/or autonomous unmanned SOLAS vessels).

Finally, it is worth saying that the traditional PB will never fade. PBs will be provided yet as paper books or pdf files extracting and rendering the entire database contents; likewise, Notice to Mariners will be sent to sailors as traditional collections of notices limiting the database extraction only to the modified database content. 